\title{
Optimal estimates for the semigroup generated by the classical Volterra operator on $L_{p}$-spaces
}

\section{Oleksandr Gomilko}

Received: 4 November 2010 / Accepted: 6 June 2011 / Published online: 29 June 2011

(C) The Author(s) 2011. This article is published with open access at Springerlink.com

Abstract Optimal upper bounds are given for the norm of the semigroup $\left(e^{-t V}\right)_{t \geq 0}$, where $V$ is the classical Volterra operator acting on $L_{p}[0,1], 1 \leq p \leq \infty$. In particular, for every $p \in[1, \infty]$ we prove that

$$
\varlimsup_{t \rightarrow+\infty}\left(t^{-|1 / 4-1 /(2 p)|}\left\|e^{-t V}\right\|_{L_{p}}\right)>0 .
$$

Keywords Volterra operator $\cdot L_{p}$-space $\cdot$ Operator semigroup

\section{Introduction}

We shall consider the classical integral Volterra operator $V$ defined on the space $L_{p}=$ $L_{p}[0,1], 1 \leq p \leq \infty$, by

$$
(V f)(x)=\int_{0}^{x} f(t) d t, \quad f \in L_{p} .
$$

The operator $V$ acts boundedly on $L_{p}$ and then the exponential function

$$
e^{z V}=\sum_{m=0}^{\infty} \frac{z^{m} V^{m}}{m !}, \quad z \in \mathbb{C},
$$

Communicated by Jerome A. Goldstein.

O. Gomilko (凶)

Nicolaus Copernicus University, Torun, Poland

e-mail: alex@gomilko.com

O. Gomilko

Institute of Mathematics, Polish Academy of Sciences, Warsaw, Poland 
is well defined.

It was proved in [1, Theorem 1.2] that

$$
\lim _{t \rightarrow+\infty} \frac{\ln \left\|e^{t e^{i \theta} V}\right\|_{L_{p}}}{t^{1 / 2}}=\sqrt{2} \cos (\theta / 2), \quad \theta \in(-\pi, \pi],
$$

where $1 \leq p \leq \infty$. For $|\theta|<\pi$ the right-hand side of the equality (2) is positive. On the other hand for $\theta=\pi$ the limit in (2) is zero. Thus, a natural and interesting case $\theta=\pi$ requires a further investigation.

As observed in [2], the semigroup $\left(e^{-t V}\right)_{t \geq 0}$ is uniformly bounded on $L_{p}$ if and only if $p=2$. Indeed, suppose that for some $p \in[1,+\infty]$ one has $\left\|e^{-t V}\right\|_{L_{p}} \leq M$, $t \geq 0$. Then by the Laplace transform representation for the generator's resolvent (see e.g. [3])

$$
\left\|(I+V)^{-n}\right\|_{L_{p}}=\left\|(-V-I)^{-n}\right\|_{L_{p}} \leq M, \quad n \in \mathbb{N} .
$$

On the other hand, by [4, Theorem 1.1] (for $p=1$ this is the result due to Hille [5]),

$$
c_{2} \leq n^{-|1 / 4-1 /(2 p)|}\left\|(I+V)^{-n}\right\|_{L_{p}} \leq c_{1}, \quad n \geq 1,
$$

for some constants $c_{1}, c_{2}>0$, and then in view of (3) we obtain that $p=2$. If $p=2$ then the operator $V$ is accretive:

$$
\operatorname{Re}(V f, f)_{L_{2}}=\frac{1}{2}\left|\int_{0}^{1} f(x) d x\right|^{2} \geq 0, \quad f \in L_{2},
$$

and therefore $\left(e^{-t V}\right)_{t \geq 0}$ is a semigroup of contractions on $L_{2}$ :

$$
\left\|e^{-t V}\right\|_{L_{2}} \leq 1, \quad t \geq 0
$$

In this note we shall prove that

$$
t^{-|1 / 4-1 /(2 p)|}\left\|e^{-t V}\right\|_{L_{p}} \leq \tilde{c}_{1}, \quad t \geq 1,
$$

and

$$
t_{n}^{-|1 / 4-1 /(2 p)|}\left\|e^{-t_{n} V}\right\|_{L_{p}} \geq \tilde{c}_{2}, \quad n \geq 1,
$$

for some positive sequence $t_{n} \rightarrow \infty, n \rightarrow \infty$, thus providing a semigroup counterpart for the estimate (4) obtained in [4].

\section{Main result}

Let $J=-V$. We have

$$
\left(J^{n} f\right)(x)=\frac{(-1)^{n}}{(n-1) !} \int_{0}^{x}(x-s)^{n-1} f(s) d s, \quad n \in \mathbb{N},
$$


and then

$$
\begin{aligned}
\left(e^{t J} f\right)(x) & =f(x)+\sum_{n=1}^{\infty} \frac{t^{n}(-1)^{n}}{(n-1) ! n !} \int_{0}^{x}(x-s)^{n-1} f(s) d s \\
& =f(x)+\int_{0}^{x} K(x-s ; t) f(s) d s,
\end{aligned}
$$

where

$$
K(z ; t):=\sum_{n=1}^{\infty} \frac{z^{n-1} t^{n}(-1)^{n}}{(n-1) ! n !}=-t \sum_{n=0}^{\infty} \frac{(-t z)^{n}}{n !(n+1) !}=-\frac{\sqrt{t}}{\sqrt{z}} J_{1}(2 \sqrt{t z})
$$

and $J_{1}(\cdot)$ is the Bessel function of the first kind and of the first order. So,

$$
\left(e^{t J} f\right)(x)=f(x)-(S(t) f)(x), \quad t>0,
$$

where

$$
\begin{aligned}
(S(t) f)(x) & =\sqrt{t} \int_{0}^{x} \frac{J_{1}(2 \sqrt{t s})}{\sqrt{s}} f(x-s) d s \\
& =\sqrt{t} \int_{0}^{x} \frac{J_{1}(2 \sqrt{t(x-s)})}{\sqrt{x-s}} f(s) d s .
\end{aligned}
$$

From (5) and (6), using Minkowski's inequality, we have

$$
\begin{aligned}
\left\|e^{t J} f\right\|_{L_{p}} & \leq\|f\|_{L_{p}}+\|f\|_{L_{p}} \sqrt{t} \int_{0}^{1} \frac{\left|J_{1}(2 \sqrt{t s})\right|}{\sqrt{s}} d s \\
& =\left(1+\int_{0}^{2 \sqrt{t}}\left|J_{1}(s)\right| d s\right) \cdot\|f\|_{L_{p}},
\end{aligned}
$$

and by the well-known estimate for Bessel functions [6, Chap. 7]:

$$
\left|J_{1}(s)\right| \leq \frac{c}{\sqrt{s}}, \quad s>0,
$$

for some $c>0$, so we obtain for some constant $M$

$$
\left\|e^{t J}\right\|_{L_{p}} \leq M t^{1 / 4}, \quad t \geq 1,1 \leq p \leq \infty .
$$

Moreover, since $\left(e^{t J}\right)_{t \geq 0}$ is a semigroup of contractions on $L_{2}$, by the Riesz-Thorin interpolation theorem [7, p. 97] and (7) we get

$$
\begin{gathered}
\left\|e^{t J}\right\|_{L_{p}} \leq\left\|e^{t J}\right\|_{L_{2}}^{2-2 / p}\left\|e^{t J}\right\|_{L_{1}}^{2 / p-1} \leq M^{2 / p-1} t^{1 /(2 p)-1 / 4}, \quad t \geq 1, p \in(1,2), \\
\left\|e^{t J}\right\|_{L_{p}} \leq\left\|e^{t J}\right\|_{L_{2}}^{2 / p}\left\|e^{t J}\right\|_{L_{\infty}}^{1-2 / p} \leq M^{1-2 / p} t^{1 / 4-1 /(2 p)}, \quad t \geq 1, p \in(2, \infty),
\end{gathered}
$$


so that

$$
\left\|e^{t J}\right\|_{L_{p}} \leq M^{|2 / p-1|} t^{|1 /(2 p)-1 / 4|}, \quad t \geq 1,1 \leq p \leq \infty .
$$

The following theorem, which is the main result of the note, states that the estimate (8) is sharp for any $p \in[1, \infty]$.

Theorem For every $p \in[1, \infty]$ :

$$
\varlimsup_{t \rightarrow+\infty}\left(t^{-|1 / 4-1 /(2 p)|}\left\|e^{t J}\right\|_{L_{p}}\right)>0 .
$$

Proof Let $q$ be the conjugate exponent of $p$, that is $1 / p+1 / q=1$. Then by $[4$, p. 765] we obtain

$$
\left\|e^{t J}\right\|_{L_{q}}=\left\|e^{t J}\right\|_{L_{p}}, \quad 1 \leq p \leq \infty .
$$

Thus is suffices to prove the estimates (9) for $p \in[1,2]$.

Let $p \in[1,2]$ be fixed. First of all, from (6) it follows that

$$
\begin{aligned}
\|S(t) f\|_{L_{p}} & \geq\|S(t) f\|_{L_{p}[1 / t, 1]} \\
& \geq\left\|S_{0}(t) f\right\|_{L_{p}[1 / t, 1]}-\left\|S_{1}(t) f\right\|_{L_{p}[1 / t, 1]}, \quad f \in L_{p}, t>1,
\end{aligned}
$$

where the operator functions $S_{j}(t), j=0,1$ are given by

$$
\begin{aligned}
& \left(S_{0}(t) f\right)(x)=\sqrt{t} \int_{0}^{x-1 / t} \frac{J_{1}(2 \sqrt{t(x-s)})}{\sqrt{x-s}} f(s) d s, \\
& \left(S_{1}(t) f\right)(x)=\sqrt{t} \int_{x-1 / t}^{x} \frac{J_{1}(2 \sqrt{t(x-s)})}{\sqrt{x-s}} f(s) d s .
\end{aligned}
$$

By Minkowski’s inequality,

$$
\begin{aligned}
\left\|S_{1}(t) f\right\|_{L_{p}[1 / t, 1]} & \leq \sqrt{t} \int_{0}^{1 / t} \frac{\left|J_{1}(2 \sqrt{t s})\right|}{\sqrt{s}}\left(\int_{1 / t}^{1}|f(x-s)|^{p} d y\right)^{1 / p} d s \\
& \leq\|f\|_{L_{p}} \sqrt{t} \int_{0}^{1 / t} \frac{\left|J_{1}(2 \sqrt{t s})\right|}{\sqrt{s}} d s \\
& =\|f\|_{L_{p}} \int_{0}^{2}\left|J_{1}(s)\right| d s=c_{0}\|f\|_{L_{p}},
\end{aligned}
$$

where the constant $c_{0}>0$ does not depend on $t>1$. So, to prove (9) it suffices to show that

$$
\varlimsup_{t \rightarrow+\infty}\left\{t^{-|1 / 4-1 /(2 p)|}\left(\sup _{f \in L_{p}} \frac{\left\|S_{0}(t) f\right\|_{L_{p}[1 / t, 1]}}{\|f\|_{L_{p}[0,1-1 / t]}}\right)\right\}>0 .
$$

We will use the following asymptotic formula for the Bessel function [6, Sect. 7.4]:

$$
J_{1}(2 \sqrt{t(x-s)})=-\frac{\cos (2 \sqrt{t(x-s)}+\pi / 4)}{\sqrt{\pi} t^{1 / 4}(x-s)^{1 / 4}}+\mathrm{O}\left(t^{-3 / 4}(x-s)^{-3 / 4}\right),
$$




$$
t(x-s) \rightarrow \infty
$$

Observe that $S_{0}(t) f$ can be decomposed as

$$
\left(S_{0}(t) f\right)(x)=-\left(S_{0,1} f\right)(x)+\left(S_{0,2}(t) f\right)(x), \quad x \in(1 / t, 1),
$$

where

$$
\left(S_{0,1} f\right)(x)=\frac{t^{1 / 4}}{\sqrt{\pi}} \int_{0}^{x-1 / t} \frac{\cos (2 \sqrt{t(x-s)}+\pi / 4)}{(x-s)^{3 / 4}} f(s) d s,
$$

and

$$
\left|\left(S_{0,2}(t) f\right)(x)\right| \leq c t^{-1 / 4} \int_{0}^{x-1 / t} \frac{|f(s)|}{(x-s)^{5 / 4}} d s=c_{1} t^{-1 / 4} \int_{1 / t}^{x} \frac{|f(x-s)|}{s^{5 / 4}} d s .
$$

Then, using Minkowski's inequality, we get the estimate

$$
\begin{aligned}
\left\|S_{0,2}(t) f\right\|_{L_{p}[1-1 / t, 1]} & \leq c_{1} t^{-1 / 4} \int_{1 / t}^{1} \frac{1}{s^{5 / 4}}\left(\int_{1-1 / t}^{1} \chi(x-s)|f(x-s)|^{p} d x\right)^{1 / p} d s \\
& \leq c_{1} t^{-1 / 4} \int_{1 / t}^{1} \frac{d s}{s^{5 / 4}}\|f\|_{L_{p}[0,1-1 / t]} \leq 4 c_{1}\|f\|_{L_{p}[0,1-1 / t]},
\end{aligned}
$$

where $\chi$ is the characteristic function of $[0,+\infty)$.

Furthermore

$$
\left\|S_{0,1}(t) f\right\|_{L_{p}[1 / t, 1]}=\frac{t^{1 / 4}}{\sqrt{\pi}}\|\tilde{S}(t) f\|_{L_{p}[0,1-1 / t]},
$$

where the operator function $\tilde{S}(t)$ is defined as

$$
(\tilde{S}(t) f)(x):=\int_{0}^{y} \frac{\cos (2 \sqrt{t(x-s+1 / t)}+\pi / 4)}{(x-s+1 / t)^{3 / 4}} f(s) d s, \quad x \in(0,1-1 / t) .
$$

Thus, by (11) and (12), we conclude that for the proof of the inequality (10), and then for the proof of the theorem, it is enough to prove that

$$
\varlimsup_{t \rightarrow+\infty}\left\{t^{-|1 / 4-1 /(2 p)|} t^{1 / 4}\left(\sup _{f \in L_{p}[0,1-1 / t]} \frac{\|\tilde{S}(t) f\|_{L_{p}[0,1-1 / t]}}{\|f\|_{L_{p}[0,1-1 / t]}}\right)\right\}>0 .
$$

Let $t_{n}=\pi^{2} n^{2}$, where $n=2,4, \ldots$ Then for $k=1,2, \ldots, n$ we have

$$
\cos \left(2 \sqrt{t_{n} z}+\pi / 4\right) \geq \frac{\sqrt{2}}{2}, \quad z \in I_{k}:=\left[\frac{(k-1 / 4)^{2}}{n^{2}}, \frac{k^{2}}{n^{2}}\right] \subset[0,1] .
$$

Note that

$$
x-s+1 / t_{n} \in I_{k}, \quad s \in[1,1 /(4 n)], x \in M_{k},
$$


where

$$
M_{k}=\left[\frac{(k-1 / 4)^{2}}{n^{2}}-\frac{1}{t_{n}}+\frac{1}{4 n}, \frac{k^{2}}{n^{2}}-\frac{1}{t_{n}}\right], \quad k=\frac{n}{2}+1, \ldots, n .
$$

The lengths $\left|M_{k}\right|$ of the segments $M_{k}$ satisfy

$$
\left|M_{k}\right|=\frac{k^{2}}{n^{2}}-\frac{(k-1 / 4)^{2}}{n^{2}}-\frac{1}{4 n}=\frac{k-n / 2-1 / 8}{2 n^{2}},
$$

so that

$$
d_{n}:=\sum_{k=n / 2+1}^{n}\left|M_{k}\right|=\frac{1}{2 n^{2}} \sum_{k=n / 2+1}^{n}(k-n / 2-1 / 8)=\frac{4 n-9}{32 n} \geq \frac{1}{9}, \quad n>20 .
$$

Let now $n>20$ be an even integer, let $g$ be the characteristic function of

$$
M=\bigcup_{k=n / 2}^{n} M_{k}
$$

and let $f$ be the characteristic function of the segment $\left[1,(4 n)^{-1}\right]$. Set $\beta_{n}=1-1 / t_{n}$. Then

$$
\begin{gathered}
\|f\|_{L_{p}\left[0, \beta_{n}\right]}=\left(\frac{1}{4 n}\right)^{1 / p} \leq \frac{1}{n^{1 / p}}, \quad\|g\|_{L_{q}\left[0,1-1 / t_{n}\right]} \leq 1, \\
\left|\int_{0}^{\beta_{n}}(\tilde{S} f)(x) g(x) d x\right| \leq\|\tilde{S} f\|_{L_{p}\left[0, \beta_{n}\right]}\|g\|_{L_{q}\left[0, \beta_{n}\right]} \leq\|\tilde{S} f\|_{L_{p}\left[0, \beta_{n}\right]}, \quad \frac{1}{p}+\frac{1}{q}=1 .
\end{gathered}
$$

On the other hand, using properties of the segments $M_{k}$ (see (14), (15)), we obtain

$$
\begin{aligned}
\left|\int_{0}^{\beta_{n}}(\tilde{S} f)(x) g(x) d x\right| & =\left|\int_{x \in M}(\tilde{S} f)(x) d x\right| \\
& =\int_{x \in M} \int_{0}^{1 / 4 n} \frac{\cos \left(2 \sqrt{t_{n}\left(x-s+1 / t_{n}\right.}+\pi / 4\right)}{\left(x-s+1 / t_{n}\right)^{3 / 4}} d s d x \\
& \geq \frac{1}{\sqrt{2}} \int_{x \in M} \int_{0}^{1 / 4 n} \frac{d s d x}{\left(x-s+1 / t_{n}\right)^{3 / 4}} \\
& \geq \frac{1}{\sqrt{2}} \int_{x \in M} \int_{0}^{1 / 4 n} d s d x=\frac{1}{\sqrt{2}} \frac{d_{n}}{4 n} \geq \frac{1}{72 n} .
\end{aligned}
$$

Thus

$$
t_{n}^{1 / 4} \frac{\|\tilde{S} f\|_{L_{p}\left[0, \beta_{n}\right]}}{\|f\|_{L_{p}\left[0, \beta_{n}\right]}} \geq \sqrt{\pi} \frac{n^{1 / p}}{72 n^{1 / 2}}=c t_{n}^{(1 / p-1 / 2) / 2}, \quad c>0 .
$$

If $p \in[1,2]$, then the last inequality implies (13), and then (9) follows. The proof is complete. 


\section{Comments and remarks}

Define the differential operator $A$ on $L_{p}, 1 \leq p<\infty$, by

$(A f)(y)=-f^{\prime}(y), \quad$ with the domain $D(A)=\left\{f \in W_{p}^{1}[0,1]: f(0)=0\right\}$,

where $W_{p}^{1}$ stands for the Sobolev space. The operator $A$ generates the nilpotent $C_{0^{-}}$ semigroup $\left(e^{t A}\right)_{t \geq 0}$ given by

$$
\left(e^{t A} f\right)(x)=f(x-t), \quad 0<x-t \leq 1, \quad\left(e^{t A} f\right)(x)=0, \quad 0 \leq x<t,
$$

and $A^{-1}=J$. So, the Theorem provides the example of a nilpotent $C_{0}$-semigroup $\left(e^{t A}\right)_{t \geq 0}$ on $L_{p}, p \in[1, \infty), p \neq 2$, such that the $C_{0}$-semigroup $\left(e^{t A^{-1}}\right)_{t \geq 0}$ is not uniformly bounded (more precisely, the norm of $e^{t A^{-1}}$ grows at infinity as $t^{\alpha_{p}}, \alpha_{p}=$ $|1 / 4-1 /(2 p)|)$.

Let us consider a more general situation. Let $X$ be a Banach space with the norm $\|\cdot\|$. Denote by $\mathcal{G}=\mathcal{G}(X)$ the set of generators of uniformly bounded $C_{0}$-semigroups on $X$ and by $\mathcal{G}_{\exp }=\mathcal{G}_{\exp }(X)$ the set of generators of exponentially stable $C_{0^{-}}$semigroup acting on $X$.

If for $A \in \mathcal{G}_{\text {exp }}(X)$ one has for some $M \geq 1, \omega>0$

$$
\left\|e^{t A}\right\| \leq M e^{-\omega t}, \quad t \geq 0,
$$

then the inverse operator $A^{-1}$ is bounded, so that $A^{-1}$ generates the $C_{0}$-semigroup $\left(e^{t A^{-1}}\right)_{t \geq 0}$ given by

$$
e^{t A^{-1}}=\sum_{m=0}^{\infty} \frac{t^{m} A^{-m}}{m !}, \quad t \geq 0 .
$$

It can also be shown that in this case (see $[8,9])$ the semigroup $\left(e^{t A^{-1}}\right)_{t \geq 0}$ has the following integral representation:

$$
e^{t A^{-1}} x=x-\sqrt{t} \int_{0}^{\infty} \frac{J_{1}(2 \sqrt{t s})}{\sqrt{s}} e^{s A} x d s, \quad t>0, x \in X
$$

(compare with (5), (6)), and, in particular, we have the estimate

$$
\left\|e^{t A^{-1}}\right\| \leq 1+M \sqrt{t} \int_{0}^{\infty} \frac{J_{1}(2 \sqrt{t s})}{\sqrt{s}} e^{-\omega s} d s \leq c t^{1 / 4}, \quad t \geq 1 .
$$

Consider now $A \in \mathcal{G}_{\exp }\left(L_{p}(\Omega, d \mu)\right), 1 \leq p<\infty$, where $(\Omega, \mu)$ is a $\sigma$-finite measure space. Suppose that operator $A \in \mathcal{G}_{\exp }\left(L_{p}\right), p \in[1, \infty)$ is such that

$$
\left\|e^{t A}\right\|_{L_{p}} \leq M e^{-\omega t}, \quad t \geq 0,
$$

for some constants $M \geq 1$ and $\omega>0$, which do not depend on $p$. Then from (17) and the Riesz-Thorin interpolation theorem, we obtain the estimates

$$
\left\|e^{t A^{-1}}\right\|_{L_{p}} \leq c_{1}\left\|e^{t A^{-1}}\right\|_{L_{2}}^{2-2 / p} t^{|1 /(2 p)-1 / 4|}, \quad t \geq 1, p \in[1, \infty) .
$$


Thus, if the semigroup $\left(e^{t A}\right)_{t \geq 0}$ is uniformly bounded on $L_{2}$, then from (18) we have

$$
\left\|e^{t A^{-1}}\right\|_{L_{p}} \leq c_{2} t^{|1 /(2 p)-1 / 4|}, \quad t \geq 1, p \in[1, \infty),
$$

and the above theorem shows the sharpness of (19) for $L_{p}(\Omega, \mu), p \in[1,2) \cup(2, \infty)$.

The problem whether the inverse of the generator of a uniformly bounded $C_{0^{-}}$ semigroup is again a generator of a uniformly bounded $C_{0}$-semigroup was posed by deLaubenfels in [10]. If $A$ is an injective linear operator on $X$ with dense range generating a uniformly bounded analytic $C_{0}$-semigroup, then it is well known that $A^{-1}$ also generates such a semigroup [10].

On the other hand, it was shown in $[8,9,11,12]$ that there exists a Banach space $X$ and an injective linear operator on $X$ with dense range generating a uniformly bounded $C_{0}$-semigroup whose inverse does not generate a $C_{0}$-semigroup. In [9] this was proved for $X=l_{p}, p \in(1,2) \cup(2, \infty)$.

If $X=H$ is an infinite-dimensional Hilbert space then the question whether the implication

$$
A \in \mathcal{G}(H), \operatorname{ker} A=\{0\} \Rightarrow A^{-1} \in \mathcal{G}(H)
$$

holds is still open.

Acknowledgement I would like to thank the referee for useful remarks and suggestions.

Open Access This article is distributed under the terms of the Creative Commons Attribution Noncommercial License which permits any noncommercial use, distribution, and reproduction in any medium, provided the original author(s) and source are credited.

\section{References}

1. Bermudo, S., Montes-Rodríguez, A., Shkarin, S.: Orbits of operators commuting with the Volterra operator. J. Math. Pures Appl. 89, 145-173 (2008)

2. Lyubich, Y.: The power boundedness and resolvent conditions for functions of the classical Volterra operator. Stud. Math. 196, 41-63 (2010)

3. Goldstein, J.A.: Semigroups of Linear Operators and Applications. Oxford University Press, New York (1985)

4. Montes-Rodríguez, A., Sánchez-Álvarez, J., Zemánek, J.: Uniform Abel-Kreiss boundedness and the extremal behaviour of the Volterra operator. Proc. Lond. Math. Soc. 91, 761-788 (2005)

5. Hille, E.: Remarks on ergodic theorems. Trans. Am. Math. Soc. 57, 246-269 (1945)

6. Erde'lyi, A., Magnus, W., Oberhettinger, F., Tricomi, F.G.: Higher Transcendental Functions, vol. 2. McGraw-Hill, New York (1953)

7. Katznelson, Y.: An Introduction to Harmonic Analysis. Dover, New York (1976)

8. Zwart, H.: Is $A^{-1}$ an infinitesimal generator. In: Perspectives in Operator Theory, Warsaw, 2004. Banach Center Publications, vol. 75, pp. 303-313. Polish Academy of Sciences, Institute of Mathematics, Warsaw (2007)

9. Gomilko, A.M., Zwart, H., Tomilov, Y.: Inverse operator of the generator of a $C_{0}$-semigroup. Mat. Sb. 198, 35-50 (2007) [in Russian]; English transl. in Sb. Math. 198, 1095-1110 (2007)

10. deLaubenfels, R.: Inverses of generators. Proc. Am. Math. Soc. 104, 443-448 (1988)

11. Komatsu, H.: Fractional powers of operators. Pac. J. Math. 19, 285-346 (1966)

12. deLaubenfels, R.: Inverses of generators of nonanalytic semigroups. Stud. Math. 191, 11-38 (2009) 